\title{
Type 2 Diabetes, Physical Activity and Risk of Mild Cognitive Impairment and Dementia in Community Dwelling Old Adults
}

\section{Eymundsdottir $\mathrm{H}^{1 *}$, Chang $\mathrm{M}^{1}$, Geirsdottir $\mathrm{OG}^{2}$, Jonsson $\mathrm{PV}^{1,3,4}$, Gudnason $\mathrm{V}^{3,5}$, Launer $\mathrm{L}^{6}$ and Ramel $\mathrm{A}^{2}$}

${ }^{1}$ The Icelandic Gerontological Research Center, the National University Hospital of Iceland, Reykjavik, Iceland

${ }^{2}$ Faculty of Food Science and Nutrition, University of Iceland, Reykjavik, Iceland

${ }^{3}$ Faculty of Medicine, University of Iceland, Iceland

${ }^{4}$ Department of Geriatrics, the National University Hospital of Iceland, Reykjavik, Iceland

${ }^{5}$ Icelandic Heart Association, Kopavogur, Iceland

${ }^{6}$ Laboratory of Epidemiology and Population Sciences, National Institute on Aging,

National Institutes of Health, Bethesda, Maryland, USA

*Corresponding Author: Eymundsdottir H, The Icelandic Gerontological Research

Center, the National University Hospital of Iceland, Reykjavik, Iceland.
Received: September 21, 2021

Published: October 21, 2021

(C) All rights are reserved by Eymundsdottir

H., et al.

\begin{abstract}
Introduction: We aim to investigate the longitudinal associations between level of physical activity (PA) and the risk of mild cognitive impairment (MCI) and dementia among type 2 diabetes (T2D) and pre-diabetes (PD) participants who are cognitively normal at study entry.

Methods: We used data from the Age-Gene/Environment-Susceptibility-Reykjavik-Study (65-96 years). From the original sample, 3001 participants with a complete evaluation of T2D, MCI and dementia were included in this analysis.

Results: During follow-up (5.2 years), 8.5\% $(n=256)$ developed MCI and 3.7\% ( $n=111)$ developed dementia. T2D participants had an increased risk for MCI $(\mathrm{OR}=1.632, \mathrm{P}=0.021)$ and both $\mathrm{PD}$ and T2D individuals had increased risk for dementia $(\mathrm{OR}=1.947, \mathrm{P}$ $=0.003$ and $\mathrm{OR}=2.101, \mathrm{P}=0.026)$. $\mathrm{PA}$ was associated with lower dementia risk in normal participants only $(\mathrm{OR}=0.611, \mathrm{P}=0.019)$.

Discussion: Older adults with PD/T2D had low level of PA and higher risk of declining cognitive function.

Keywords: Type 2 Diabetes; Mild Cognitive Impairment; Dementia; Physical Activity; Glucose Regulations

\section{Introduction}

Diabetes has been increasing worldwide [1] with increasing prevalence associated with age, making older adults frequently affected [2]. Type 2 diabetes (T2D) accounts for the majority of diabetes patients ( $85 \%$ to $90 \%$ ), characterized by a worsening insulin sensitivity finally resulting into hyperglycemia [3] being a serious cause of cardiovascular disease, kidney disease and neuropathy [4]. Studies have suggested that old adults with T2D have a higher

risk ( $>50 \%$ ) for developing cognitive deficits, especially when glycemic control is poor [5-7].

Regular physical activity (PA) can have positive effects for glucose regulations especially in patients with T2D [8,9], and recent studies have indicated that PA can improve cognition in old adults as well $[10,11]$. Epidemiological studies often associated physical exercise with improved cognitive function [6], decreased hazards
\end{abstract}


of dementia and fewer ageing associated alterations in the brain $[12,13]$. Data from brain imaging studies indicate that physical activity can prevent brain atrophy in old populations and experimental evidence suggests that resistance training also positively effects cognition in this age group [14,15]. Additionally, PA can improve glucose regulations and cardiovascular risk factors which all represent a threat for good cognitive function when compromised [1618].

Despite that the evidence is solid that PA is related to good cognitive function, majority of research has been carried out in healthy older adults. Current literature is less clear whether the positive findings on PA and cognitive function can be applied to old adults with poor glucose regulations or diabetes [19]. A recently published systematic review investigating the relationship between exercise and cognitive function in participants with diabetes or poor glucose regulations were only few of the cognitive outcomes were significant [16].

Thus, the present cohort study investigated T2D, PA and risk of MCI and dementia during 5.2 years of follow-up in community dwelling old Icelandic adults.

The specific aims of the present study were to investigate: the MCI- and dementia risk in healthy, pre-diabetic and diabetic participants; whether lower PA and poorer lifestyle in diabetic participants explain increased risk of MCI or dementia; whether diabetic status modifies the ability of PA to prevent MCI or dementia.

\section{Methods}

\section{Study population and study design}

This longitudinal analysis is based on data from the AGES-Reykjavik study ( $N=5764$ ) enrolled in 2002-2006 as a continuation of the population-based Reykjavik Study (RS) in Iceland, initiated in 1967. Detailed baseline information have been described in a previous AGES-study paper [20]. Between 2007-2011, AGES I participants (58\%, $\mathrm{N}=3316)$ returned to a second examination, a 5-year follow-up visit (AGES II). The current study included participants who were cognitively normal at baseline examination and had the relevant follow-up examination including a cognitive test battery ( $\mathrm{N}=3001)$. The study was approved by the National Bioethics Committee in Iceland (approval VSN-00-063), the Data Protection Authority and by the National Institute on Aging Intramural Institutional Review Board. Written informed consent was obtained from all participants.

\section{Anthropometrics}

Weight and height were measured and BMI was calculated as $\mathrm{kg} /$ $\mathrm{m}^{2}$.

\section{Mild cognitive impairment and Dementia}

The criterion for MCI diagnosis was having deficits in memory or one other domain of cognitive function or deficits in at least 2 cognitive domains without being severe enough to cross the threshold for dementia and without loss of instrumental activities of daily living [21].

Assessment of dementia was done following a three-step protocol and according to international guidelines from the Diagnostic and Statistical Manual of Mental Disorders, Fourth Edition [22]. First, the digit symbol substitution test (DSST) [23] and the MiniMental State Examination (MMSE) [24] were administered to the total sample. Participants who scored 23 or lower on the MMSE or had a raw score of 17 or lower on the DSST were administered a second diagnostic cognitive test battery. Participants who scored 8 or more on Trails B [25] (ratio of time taken for "Trails B/Trails $A^{\prime \prime}$ ) or had lower than total score of 19 for the four immediate recall trials of the Rey Auditory Verbal Learning [26] went on to a third step. This step included a neurological test and a proxy interview regarding medical history, social, cognitive, and daily functioning changes of the participant.

\section{Diabetes mellitus and pre-diabetes}

Participants were categorized into being type 2 diabetic (diagnosed either as fasting serum glucose of $\geq 126 \mathrm{mg} / \mathrm{dL}$, self-reported diabetes and/or use of diabetes medication), pre-diabetic (fasting blood glucose $\geq 100$ to $<126 \mathrm{mg} / \mathrm{dL}$ ) [27] and normal health (neither of above definitions).

\section{Covariates}

\section{Demographic and lifestyle data}

Participants were asked about their age, gender, smoking habits (current smoking yes or no), alcohol consumption (shown as g/ week). Physical activity was assessed by a self-reported questionnaire. Participants were asked, how many hours per week they participated in moderate intensity PA in the past 12 months. Predefined answer categories were never, rarely, weekly but $<1$ hour 
per week, 1-3 hours per week, 4-7 hours per week and more than 7 hours per week. In statistical analyses PA categories were combined ( $\leq 3 \mathrm{~h}$ vs. $>3 \mathrm{~h}$ ). Education was categorized into two levels (elementary school or high school vs. undergraduate or more than undergraduate education). Participants were instructed in advance to bring all medication they had used during the preceding two weeks before the clinic visit.

\section{Laboratory data}

The accredited IHA laboratory performed 250HD measurements using unfrozen serum samples and the Liaison chemiluminescence immunoassay (DiaSorin Inc, Stillwater, Minnesota). Existing serum 250HD levels were then standardized [28]. Glucose levels in a capillary blood sample were estimated by the Hoffman ferricyanide method, adapted to the Technicon-Method N-9a $[29,30]$. Glucose was measured on a Hitachi 912, using reagents from Roche Diagnostics following the manufacturer's instructions. Insulin was measured with a Roche Elecsys 2010 instrument [31].

\section{Apolipoprotein E genotype}

The final sample in current study were all genotyped for APOE $\varepsilon 4$ alleles using standard methods [32]. Participants were considered APOE $\varepsilon 4$ positive if they carried $\varepsilon 3 / 4$, and $\varepsilon 4 / 4$ genotype otherwise if they carried $\varepsilon 2 / 2, \varepsilon 2 / 3$ and $\varepsilon 3 / 3$ they were considered APOE $\varepsilon 4$ non-carriers.

\section{Statistical analysis}

Statistical analyses were carried out using IBM SPSS version 26.0 (SPSS, Chicago, IL, USA). Baseline characteristics of the participants categorized by diabetic status are shown in table 1 . We used chi-square test for categorical variables and after visual inspection of the distribution and calculation of variances, we used ANOVA or Kruskal Wallis test for continuous variables to test for statistical differences. As distributions were not normal and variances between the three categories of participants were not similar for alcohol, number of medicines, glucose and insulin, we used Kruskal Wallis test.

To calculate whether diabetes status predicts onset of MCI or dementia (Table 2) logistic regression analyses were applied controlling for various confounders. For each outcome variable the following 3-step model was applied: Model 1 adjusted for age and gender; model 2 additionally adjusted for smoking, education and BMI; model 3 additionally adjusted for 250HD, PA and APOE $\varepsilon 4$.

\begin{tabular}{|c|c|c|c|c|}
\hline & Normal & $\begin{array}{c}\text { Pre- } \\
\text { diabetic }\end{array}$ & Diabetic & \\
\hline & $(n=1570)$ & $(n=1124)$ & $\begin{array}{l}(n= \\
307)\end{array}$ & \\
\hline & Mean \pm SD & Mean \pm SD & $\begin{array}{c}\text { Mean } \pm \\
\text { SD }\end{array}$ & P-value* \\
\hline Age (years) & $75.3 \pm 4.9$ & $74.6 \pm 4.9$ & $\begin{array}{c}75.1 \pm \\
4.6\end{array}$ & $<0.001$ \\
\hline Women (in \%) & 64.5 & 53.0 & 46.7 & $<0.001$ \\
\hline Smoking (yes in\%) & 9.1 & 7.8 & 6.6 & 0.218 \\
\hline $\begin{array}{l}\text { Higher education } \\
\text { (in \%) }\end{array}$ & 28.4 & 27.2 & 29.0 & 0.721 \\
\hline Alcohol (g/week) & $\begin{array}{c}14.7 \pm 31.7 \\
3.5 \pm 2.7\end{array}$ & $\begin{array}{c}18.7 \pm 37.6 \\
3.5 \pm 2.6\end{array}$ & $\begin{array}{c}15.4 \pm \\
35.3\end{array}$ & $\begin{array}{r}0.040 \\
<0.001\end{array}$ \\
\hline $\begin{array}{l}\text { Number of } \\
\text { medications }\end{array}$ & $26.3 \pm 4.0$ & $28.1 \pm 4.2$ & $5.5 \pm 3.0$ & $<0.001$ \\
\hline BMI $\left(\mathrm{kg} / \mathrm{m}^{2}\right)$ & 16.5 & 29.5 & $\begin{array}{c}29.1 \pm \\
4.5\end{array}$ & $<0.001$ \\
\hline Obese (yes in \%) & 35.2 & 32.2 & & 0.011 \\
\hline $\begin{array}{l}\text { Physical activity (no } \\
\text { in \%) }\end{array}$ & $\begin{array}{c}16.2 \\
58.9 \pm 17.3\end{array}$ & $\begin{array}{c}14.4 \\
58.1 \pm 17.8\end{array}$ & $\begin{array}{l}39.8 \\
28.2\end{array}$ & $<0.001$ \\
\hline $\begin{array}{l}\text { Physical actvity > } \\
\text { 3h/week (\%) }\end{array}$ & $93.0 \pm 5.0$ & $107.4 \pm 6.0$ & 8.5 & $<0.001$ \\
\hline 250HD (nmol/L) & $8.5 \pm 5.6$ & $13.8 \pm 7.5$ & $\begin{array}{c}55.0 \pm \\
16.7\end{array}$ & $<0.001$ \\
\hline Glucose $(\mathrm{mg} / \mathrm{dL})^{* *}$ & $27.3 \pm 2.5$ & $27.2 \pm 2.4$ & $140.5 \pm$ & 0.214 \\
\hline Insulin $(\mathrm{mU} / \mathrm{L})^{* *}$ & 8.5 & 8.2 & 35.9 & 0.028 \\
\hline $\begin{array}{l}\text { MMSE (baseline } \\
\text { score) }\end{array}$ & 3.2 & 4.7 & $\begin{array}{c}15.3 \pm \\
9.4\end{array}$ & 0.040 \\
\hline $\begin{array}{l}\text { MCI during } \\
\text { follow-up }(\%)\end{array}$ & & & $\begin{array}{c}27.1 \pm \\
2.6\end{array}$ & \\
\hline $\begin{array}{l}\text { Dementia during } \\
\text { follow-up (\%) }\end{array}$ & & & $\begin{array}{r}13.1 \\
5.9 \\
\end{array}$ & \\
\hline
\end{tabular}

Table 1: Characteristics of AGES-Reykjavik participants according to diabetic status.

Abbreviations: BMI: Body Mass Index; 250HD: 25 hydroxyvitamin D; MMSE: Mini-Mental State Exam; MCI: Mild Cognitive Impairment.

*Based on chi-square test for categorical variables and ANOVA for continuous variables. P-values for alcohol, number of medicines, glucose and insulin are based on Kruskal Wallis test.

${ }^{\dagger}$ Mean follow-up time 5.2 years. 


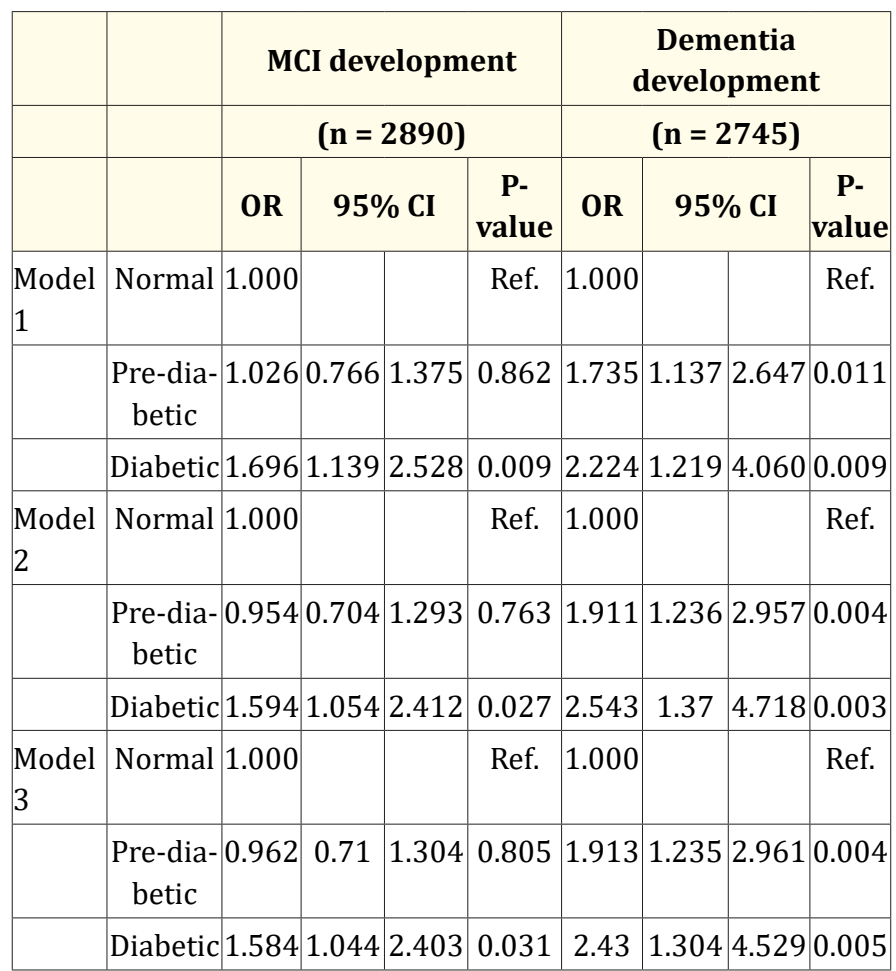

Table 2: Risk* of MCI and dementia during follow-up in normal, pre-diabetic and diabetic participants.

Abbreviations: MCI: Mild Cognitive Impairment; OR: Odds Ratio; CI: Confidence Interval.

*Based on logistic regression

Model 1: Corrected for age and gender

Model 2: Additionally, corrected for BMI, smoking and education.

Model 3: Additionally, corrected for physical activity, 250HD and APOE $\varepsilon 4$.

To calculate whether the risk of MCI or dementia diagnosis can be minimized among diabetic participants through physical activity, data were stratified by diabetic status and then logistic regression models were used. The outcome variables were MCI and dementia; the main independent variable was PA. In model 1 we adjusted for sex; in model 2 we additionally adjusted for age (Table 3).

The level of statistical significance was set at $\mathrm{p}<0.05$.

\section{Results}

The characteristics of the participants categorized by diabetic status are shown in table 1. Participants with diabetes were more often men, had higher baseline BMI, lower vitamin D levels, used more medication and were less physical active. Education, smoking and MMSE were not significantly different between the categories.

Of 3001 participants, 256 (8.5\%) developed MCI and 111 $(3.7 \%)$ developed dementia during a mean follow-up of 5.2 years. Tables 2 shows the results from logistic regression models estimating the MCI- and dementia risk for pre-diabetic and diabetic participants compared to normal participants. The calculations show an increased MCI risk for diabetic participants which was largely independent from lifestyle associated covariates or education. We also found an increased risk for dementia in both pre-diabetic and diabetic individuals when compared to normal individuals, in fully adjusted model.

The effect of the interaction between physical activity and diabetes on cognitive function outcome was not significant $\left(\mathrm{P}_{\text {interaction }}>\right.$ 0.05 for all, supplementary table 1 ). Table 3 shows the associations between PA and MCI/dementia risk stratified by diabetes status and adjusted for gender. Physical activity at baseline was associated with lower MCI risk during follow-up in normal and pre-diabetic (borderline) participants only, but not in diabetic (model 1). Part of this association was driven by age and corresponding adjustment weakened the statistical significance (model 2).

Physical activity was also associated with lower dementia risk in normal participants only, but not in pre-diabetic and diabetic participant (table 3, model 1). Here also, part of this association was driven by age and statistical significance disappeared after corresponding statistical adjustment (table 3, model 2).

\section{Discussion}

In this cohort study diabetic participants had higher MCI and dementia risk during five years of follow-up, and pre-diabetic subjects had higher dementia risk, when compared to subjects with normal glucose metabolism at baseline. Our study suggests that lower PA in diabetic participants did not explain their increased risk of MCI or dementia. The results indicate that PA was protective for MCI- and dementia risk in normal, but neither in pre-diabetic nor in diabetic participants. 
Type 2 Diabetes, Physical Activity and Risk of Mild Cognitive Impairment and Dementia in Community Dwelling Old Adults

\begin{tabular}{|c|c|c|c|c|c|c|c|c|c|c|}
\hline & & & \multicolumn{4}{|c|}{ MCI development } & \multicolumn{4}{|c|}{ Dementia development } \\
\hline & \multirow{3}{*}{$\begin{array}{c}\text { Stratification } \\
\text { Normal } \\
\end{array}$} & \multirow{3}{*}{$\begin{array}{c}\text { Independent } \\
\text { variable }\end{array}$} & \multicolumn{4}{|c|}{$(n=2890)$} & \multicolumn{4}{|c|}{$(n=2745)$} \\
\hline & & & \multirow{2}{*}{$\begin{array}{c}\text { OR } \\
0.499\end{array}$} & \multicolumn{2}{|c|}{$95 \% \mathrm{CI}$} & \multirow{2}{*}{$\begin{array}{c}\text { P-value } \\
<0.001\end{array}$} & \multirow{2}{*}{$\begin{array}{c}\text { OR } \\
0.553\end{array}$} & \multicolumn{2}{|c|}{$95 \% \mathrm{CI}$} & \multirow{2}{*}{$\begin{array}{r}\text { P-value } \\
0.049\end{array}$} \\
\hline Model 1 & & & & 0.347 & 0.718 & & & 0.306 & 0.997 & \\
\hline & Pre-diabetic & physical activity & 0.658 & 0.424 & 1.021 & 0.062 & 0.664 & 0.373 & 1.183 & 0.165 \\
\hline & Diabetic & physical activity & 0.999 & 0.502 & 1.987 & 0.996 & 0.825 & 0.300 & 2.270 & 0.702 \\
\hline \multirow[t]{3}{*}{ Model 2} & Normal & physical activity & 0.611 & 0.420 & 0.891 & 0.019 & 0.737 & 0.401 & 1.353 & 0.399 \\
\hline & Pre-diabetic & physical activity & 0.832 & 0.527 & 1.314 & 0.441 & 0.864 & 0.475 & 1.573 & 0.633 \\
\hline & Diabetic & physical activity & 1.145 & 0.558 & 2.348 & 0.717 & 0.878 & 0.314 & 2.456 & 0.749 \\
\hline
\end{tabular}

Table 3: Risk* of MCI and dementia in normal, pre-diabetic and diabetic participants depending on physical activity.

Abbreviations: MCI: Mild Cognitive Impairment; OR: Odds Ratio; CI: Confidence Interval.

*Based on stratified logistic regression

tas compared to no physical activity

Model 1: Corrected for gender

Model 2: Corrected for gender and age

\begin{tabular}{|c|c|c|c|c|c|c|c|c|c|}
\hline \multirow[b]{3}{*}{ Model 1} & \multirow{3}{*}{$\begin{array}{c}\text { Interaction } \\
\text { Physical } \\
\text { activity*Diabetic } \\
\text { status }\end{array}$} & \multicolumn{4}{|c|}{ MCI development } & \multicolumn{4}{|c|}{ Dementia development } \\
\hline & & \multirow{2}{*}{$\begin{array}{c}\text { OR } \\
0.991\end{array}$} & \multicolumn{2}{|c|}{$95 \%$ CI } & \multirow{2}{*}{$\begin{array}{c}\text { P-value } \\
0.892\end{array}$} & \multirow{2}{*}{$\begin{array}{c}\mathbf{O R} \\
1.021\end{array}$} & \multicolumn{2}{|c|}{$95 \% \mathrm{CI}$} & \multirow{2}{*}{$\begin{array}{c}\text { P-value } \\
0.843\end{array}$} \\
\hline & & & 0.865 & 1.134 & & & 0.834 & 1.249 & \\
\hline Model 2 & $\begin{array}{c}\text { Physical } \\
\text { activity*Diabetic } \\
\text { status }\end{array}$ & 0.981 & 0.856 & 1.124 & 0.781 & 0.992 & 0.811 & 1.212 & 0.936 \\
\hline
\end{tabular}

Supplementary Table 1: Interaction ${ }^{\dagger}$ between physical activity and diabetic status.

Abbreviations: MCI: Mild Cognitive Impairment; OR: Odds Ratio; CI: Confidence Interval.

†Based on logistic regression

Model 1: Corrected for gender.

Model 2: Corrected for gender and age.

Disturbed glucose levels have been associated with an elevated risk of poor cognitive function as well as dementia when compared to healthy subjects $[33,34]$. According to meta-analyses $[35,36]$ the risk for dementia diagnosis is increased by $51-73 \%$ in diabetics and our own results show somewhat higher odds between +110 to $147 \%$ depending on the degree of statistical correction. Furthermore, studies also show an increased risk for MCI in diabetic subjects (+21\%) [35] which is slightly lower than observed in our study.

There are only few studies available in literature investigating the effects of PA on cognition in participants with diabetes or disturbed glucose regulations and none of these studies have shown reduced risk from PA [19,37-41]. There are several possible explanations why PA has not been found beneficial in the prevention of 
dementia in previous studies. Some cohorts consist of rather young subjects, still showing good cognitive performance at the end of the research and in intervention studies the follow-up time might be too short to produce meaningful changes in cognition.

In the present observational study, PA levels were generally low with the lowest proportion among T2D subjects and PA was categorized into " $\leq 3 \mathrm{~h}$ " vs." $>3 \mathrm{~h}$ ". Although a more detailed categorization of PA was possible in our data, too few cases of dementia and MCI were in the single categories undermining meaningful statistical analysis. It is therefore interesting to speculate whether a higher level of PA in conjunction with a good glycemic control is necessary to maintain cognition during ageing.

In contrast to the currently available weak evidence of PA and the prevention of cognitive decline in individuals with disturbed glucose regulations, it should be mentioned that studies have shown that increased physical activity has clear beneficial physiological and psychological effects for older adults with T2D [42].

\section{Limitation}

Results of this study may be limited since categorization of major subtypes of dementia such as vascular dementia were not available and participation in PA among T2D participants might have been too low to detect the protective levels of PA.

It is a further limitation of this study that we used physical activity as a dichotomous variable which lead to a loss of information. Available data on physical activity were based on predefined answer categories beyond our control: never, rarely, weekly but $<1$ hour per week, 1-3 hours per week, 4-7 hours per week and more than 7 hours per week. PA categories were the further combined $(\leq$ $3 \mathrm{~h}$ vs. $>3 \mathrm{~h}$ ) for statistical reasons as very few participants were physical active $>7$ hours/week.

\section{Conclusion}

Our study showed that that community dwelling old adults with T2D had higher dementia and MCI risk during follow-up and pre-diabetic subjects had higher dementia risk, when compared to community dwelling old adults with normal glucose levels. In contrast to our expectations, PA was not protective from MCI and dementia neither in pre-diabetic nor in type 2 diabetic participants.

\section{Conflict of Interest}

The authors declare no conflict of interest.

\section{Acknowledgments}

This work was supported by The Foundation of St. Josef's Hospital in cooperation with The Icelandic Gerontological Research Center, National University. The funding sources did not have any role in the study design, conduct of the study, analysis of the data, or manuscript preparation.

\section{Bibliography}

1. Shaw JE., et al. "Global estimates of the prevalence of diabetes for 2010 and 2030". Diabetes Research and Clinical Practice 87.1 (2010): 4-14.

2. Rathmann W and Giani G. "Global prevalence of diabetes: estimates for the year 2000 and projections for 2030". Diabetes Care 27.10 (2004): 2568-2569. author reply 69.

3. American Diabetes A. Classification and diagnosis of diabetes". Diabetes Care 38 (2015): S8-S16.

4. Zhao Y., et al. "Prevalence of other diabetes-associated complications and comorbidities and its impact on health care charges among patients with diabetic neuropathy". Journal of Diabetes and Its Complications 24.1 (2010): 9-19.

5. Cukierman-Yaffe T., et al. "Relationship between baseline glycemic control and cognitive function in individuals with type 2 diabetes and other cardiovascular risk factors: the action to control cardiovascular risk in diabetes-memory in diabetes (ACCORD-MIND) trial”. Diabetes Care 32.2 (2009): 221-226.

6. Strachan MW., et al. "Cognitive function, dementia and type 2 diabetes mellitus in the elderly". Nature Review on Endocrinology 7.2 (2011): 108-114.

7. Cooray G., et al. "Effects of intensified metabolic control on CNS function in type 2 diabetes". Psychoneuroendocrinology 36.1 (2011): 77-86.

8. Diabetes Prevention Program Research. G. "The diabetes prevention program (DPP): description of lifestyle intervention". Diabetes Care 25.12 (2002): 2165-2171.

9. Hwang $\mathrm{MH}$ and Kim S. "Type 2 diabetes: endothelial dysfunction and exercise". Journal of Exercise Nutrition and Biochemistry 18.3 (2014): 239-247. 
10. Ahlskog JE., et al. "Physical exercise as a preventive or diseasemodifying treatment of dementia and brain aging". Mayo Clinic Proceedings 86.9 (2011): 876-884.

11. Colcombe S and Kramer AF. "Fitness effects on the cognitive function of older adults: a meta-analytic study". Psychology Science 14.2 (2003): 125-130.

12. Etgen T., et al. "Physical activity and incident cognitive impairment in elderly persons: the INVADE study". Archives of Internal Medicine 170.2 (2010): 186-193.

13. Larson EB., et al. "Exercise is associated with reduced risk for incident dementia among persons 65 years of age and older". Annals of Internal Medicine 144.2 (2006): 73-81.

14. Liu-Ambrose T., et al. "Resistance training and functional plasticity of the aging brain: a 12-month randomized controlled trial". Neurobiology Aging 33.8 (2012): 1690-1698.

15. Nagamatsu LS., et al. "Resistance training promotes cognitive and functional brain plasticity in seniors with probable mild cognitive impairment". Archives of Internal Medicine 172.8 (2012): 666-668.

16. Gasparini L and Potential XH. "Roles of insulin and IGF-1 in Alzheimer's disease". Trends in Neuroscience 26.8 (2003): 404406.

17. Helzner EP., et al. "Contribution of vascular risk factors to the progression in Alzheimer disease". Archives of Neurology 66.3 (2009): 343-348.

18. Eymundsdottir H., et al. "Serum 25-hydroxy vitamin D, physical activity and cognitive function among older adults". Journal of Aging Research and Clinical Practice 7 (2018): 143-148.

19. Zhao RR., et al. "Exercise or physical activity and cognitive function in adults with type 2 diabetes, insulin resistance or impaired glucose tolerance: a systematic review". European Review of Aging and Physical Activity 15 (2018): 1.

20. Harris TB., et al. "Age, Gene/Environment Susceptibility-Reykjavik Study: multidisciplinary applied phenomics". American Journal of Epidemiology 165.9 (2007): 1076-1087.

21. McKhann GM., et al. "The diagnosis of dementia due to Al- zheimer's disease: recommendations from the National Institute on Aging-Alzheimer's Association workgroups on diagnostic guidelines for Alzheimer's disease". Alzheimers Dementia 7.3 (2011): 263-269.

22. Sheehan DV., et al. "The Mini-International Neuropsychiatric Interview (M.I.N.I.): the development and validation of a structured diagnostic psychiatric interview for DSM-IV and ICD10". Journal of Clinical Psychiatry 59 (1997): 22-33 quiz 34-57.

23. Wechsler DW. "Adult Intelligence Scale". Manual. New York: Psychological Corporation (1955).

24. Folstein MF., et al. "“Mini-mental state”. A practical method for grading the cognitive state of patients for the clinician". Journal of Psychiatric Research 12.3 (1975): 189-198.

25. Reiten RM. "Validity of the trail making test as an indicator of organic brain damage". Perceptual and Motor Skills 8 (1958): 271-276.

26. Andrés R. L' examen clinique en psychologie. Paris: Presses universitaires de France (1964).

27. American Diabetes Association.

28. Binkley N and Sempos CT. "Vitamin D Standardization Program (VDSP). Standardizing vitamin D assays: the way forward". Journal of Bone and Mineral Research 29.8 (2014): 1709-1714.

29. Harris TB., et al. "Age, Gene/environment susceptibility-Reykjavik Study: multidisciplinary applied phenomics". American Journal of Epidemiology 165 (2007): 1076-1087.

30. Jonsdottir LS., et al. "Do lipids, blood pressure, diabetes, and smoking confer equal risk of myocardial infarction in women as in men? The Reykjavik Study". Journal of Cardiovascular Risk 9 (2002): 67-76.

31. Olafsdottir E., et al. "Unfavourable risk factors for type 2 diabetes mellitus are already apparent more than a decade before onset in a population-based study of older persons: from the Age, Gene/Environment Susceptibility-Reykjavik Study (AGES-Reykjavik)". European Journal of Epidemiology 24.6 (2009): 307-314.

Citation: Eymundsdottir H., et al. "Type 2 Diabetes, Physical Activity and Risk of Mild Cognitive Impairment and Dementia in Community Dwelling Old Adults". Acta Scientific Nutritional Health 5.11 (2021): 59-66. 
32. Eiriksdottir G., et al. "Apolipoprotein E genotype and statins affect CRP levels through independent and different mechanisms: AGES-Reykjavik Study". Atherosclerosis 186.1 (2006): $222-224$.

33. Luchsinger JA. "Type 2 diabetes, related conditions, in relation and dementia: an opportunity for prevention?" Journal of Alzheimer's Disease 20.3 (2010): 723-736.

34. Biessels GJ., et al. "Cognition and diabetes: a lifespan perspective”. Lancet Neurology 7.2 (2008): 184-190.

35. Gudala K., et al. "Diabetes mellitus and risk of dementia: A meta-analysis of prospective observational studies". Journal of Diabetes Investigation 4.6 (2013): 640-650.

36. Cheng G., et al. "Diabetes as a risk factor for dementia and mild cognitive impairment: a meta-analysis of longitudinal studies". Internal Medical Journal 42.5 (2012): 484-491.

37. Devore EE., et al. "Physical activity levels and cognition in women with type 2 diabetes". American Journal of Epidemiology 170.8 (2009): 1040-1047.

38. Yanagawa M., et al. "Association between improvements in insulin resistance and changes in cognitive function in elderly diabetic patients with normal cognitive function". Geriatrics and Gerontology International 11.3 (2011): 341-347.

39. Lehtisalo J., et al. "Association of Long-Term Dietary fat Intake, exercise, and weight with later cognitive function in the Finnish diabetes prevention study". The Journal of Nutrition, Health and Aging 20.2 (2016): 146-154.

40. Espeland MA., et al. "Impact of a Multidomain Intensive Lifestyle Intervention on Complaints About Memory, ProblemSolving, and Decision-Making Abilities: The Action for Health in Diabetes Randomized Controlled Clinical Trial". Journals of Gerontology, Series A: Biological Sciences 73.11 (2018): 15601567.

41. Rapp SR., et al. "Effect of a Long-Term Intensive Lifestyle Intervention on Cognitive Function: Action for Health in Diabetes Study". Journal of the American Geriatrics Society 65.5 (2017): 966-972.
42. Jenkins DW., et al. "Exercise and Diabetes: A Narrative Review". Journal of Foot and Ankle Surgery 56 (2017): 968-974.

Volume 5 Issue 11 November 2021 (C)All rights are reserved by Eymundsdottir H., et al. 